\title{
REVIEW
}

\section{Current and Future Considerations for the Treatment of Hospital-Acquired Pneumonia}

Philippe Montravers · Adela Harpan · Elise Guivarch

To view enhanced content go to www.advancesintherapy.com Received: January 4, 2016/Published online: February 9, 2016

(C) The Author(s) 2016. This article is published with open access at Springerlink.com

\begin{abstract}
Hospital-acquired pneumonia (HAP) and health-care-associated pneumonia (HCAP) are leading causes of death, morbidity, and resource utilization in hospitalized patients, and are associated with a broad range of Gram-positive and Gram-negative pathogens. Here, we discuss the different definitions of HAP and HCAP, review current guidelines regarding the treatment of these conditions, highlight the shortcomings of current therapeutic options, and discuss new antibiotic treatments. To optimize therapeutic outcomes in patients with HAP/HCAP, initial antimicrobial treatment must be appropriate and should be given as soon as possible; inappropriate or
\end{abstract}

Electronic supplementary material The online version of this article (doi:10.1007/s12325-016-0293-x) contains supplementary material, which is available to authorized users.

P. Montravers $(\bowtie) \cdot$ A. Harpan · E. Guivarch Département d'Anesthésie-Réanimation, CHU Bichat Claude-Bernard-HUPNVS, Assistance Publique-Hôpitaux de Paris, University Denis Diderot, PRESS Sorbonne Cité, 46 Rue

Henri-Huchard, 75018 Paris, France

e-mail: philippe.montravers@aphp.fr

P. Montravers · E. Guivarch

University Denis Diderot, PRESS Sorbonne Cité,

Paris, France delayed therapy greatly increases morbidity and mortality. Selection of the most appropriate antimicrobial agent depends on the causative pathogen(s); initial broad-spectrum therapy is commonly recommended and should cover all pathogens that may be present. Treatment selection should also take into consideration the following factors: knowledge of underlying local risk factors for antimicrobial resistance, disease staging, and risk factors related to specific pathogens such as Pseudomonas aeruginosa, Acinetobacter spp., and methicillinresistant Staphylococcus aureus (MRSA). Guidelines consistently emphasize the importance of treating HAP and HCAP with early and appropriate broad-spectrum antibiotics, and recent developments in this field have resulted in the availability of several additional treatment options. Telavancin shows potent activity against Gram-positive bacteria including MRSA and can be administered once daily; it was approved in the USA and European Union for the treatment of HAP after demonstrating non-inferiority to vancomycin. Ceftobiprole medocaril exhibits rapid antimicrobial activity against a broad range of both Gram-positive and Gram-negative 
pathogens, including MRSA. It was approved for the treatment of HAP (excluding ventilatorassociated pneumonia) and community-acquired pneumonia in Europe in 2013. These new treatments may offer effective alternative therapeutic options for the management of HAP. Funding: Basilea Pharmaceutica Ltd., Basel, Switzerland.

Keywords: Antimicrobial Ceftobiprole; Hospital-acquired Health-care-associated resistance; pneumonia; pneumonia; Monotherapy; MRSA; Multidrug resistance; Staphylococcus aureus; Tedizolid; Telavancin

\section{INTRODUCTION}

Hospital-acquired pneumonia (HAP) is one of the most common nosocomial infections, occurring at a rate of 5 to more than 20 cases per 1000 hospital admissions [1, 2], and is a leading cause of death, morbidity, and resource utilization in hospitalized patients [2-6]. Health-care-associated pneumonia (HCAP) describes pneumonia contracted as a result of contact with health-care settings other than hospitals, such as assisted living centers and rehabilitation facilities. A large, multicenter study in the USA found that $>20 \%$ of 4543 patients hospitalized with pneumonia had HCAP, with mortality similar to that for HAP (19.8\% vs. $18.8 \%$ ) [7].

This review discusses the different definitions of HAP and HCAP, reviews current guidelines regarding the treatment of patients with these conditions, and highlights the shortcomings of current therapeutic options. Antibiotics recently approved for the treatment of HAP (telavancin and ceftobiprole) together with tedizolid (currently in clinical development) are also discussed; new agents approved for the treatment of ventilatorassociated pneumonia (VAP) are not considered here as this is beyond the scope of this review. This article is based on previously conducted studies and does not involve any new studies of human or animal subjects performed by any of the authors.

\section{DEFINITIONS OF HAP AND HCAP}

In the USA and Europe, HAP is defined as pneumonia that develops $48 \mathrm{~h}$ or more after hospital admission $[2,8,9]$. HAP is distinct from VAP, which arises more than $48-72 \mathrm{~h}$ after endotracheal intubation [2]. Many patients with HAP are treated outside the intensive care unit (ICU) and do not require mechanical ventilation [10]. HCAP is a more recent term than HAP and its clinical relevance remains disputed. HCAP was recognized in the American Thoracic Society (ATS) 2005 guidelines, which describe a distinct form of pneumonia not contracted during a hospital admission, but which resembles HAP more than community-acquired pneumonia (CAP; see Table 1 for a full definition of HCAP) [2]. Since the publication of the ATS guidelines there has been continued debate about the clinical relevance of HCAP, and indeed, the term HCAP may not be retained in the forthcoming updated ATS guidelines. Joint European guidelines on the management of HAP, published in 2009 by the European Respiratory Society (ERS), the European Society of Clinical Microbiology and Infectious Diseases (ESCMID), and the European Society of Intensive Care Medicine (ESICM) call for HCAP to be recognized as a distinct disease from CAP, but do not provide a specific definition [11]. However, separate guidelines issued by the ERS and ESCMID in 2011 on the management of lower respiratory tract infections (excluding 


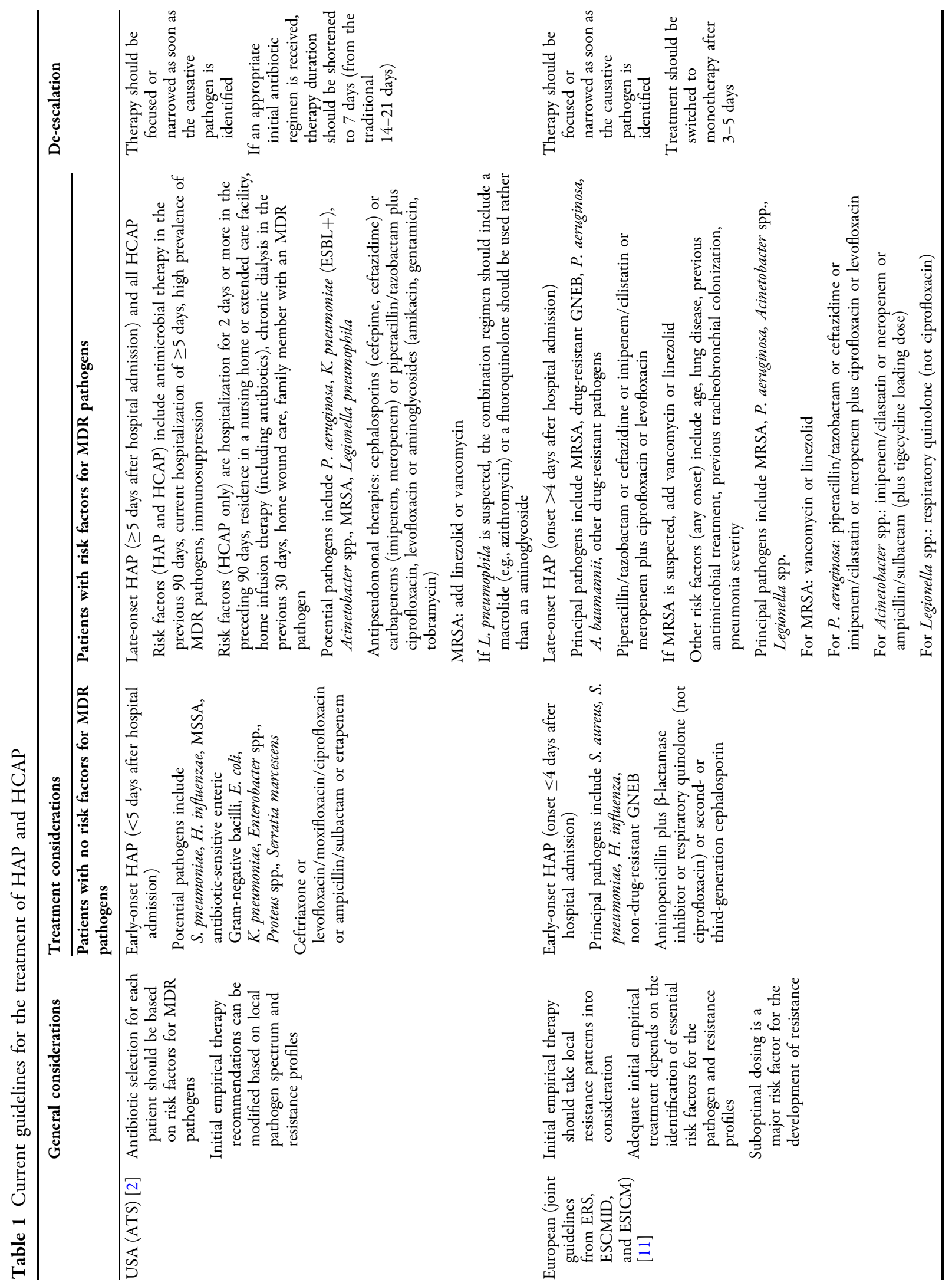




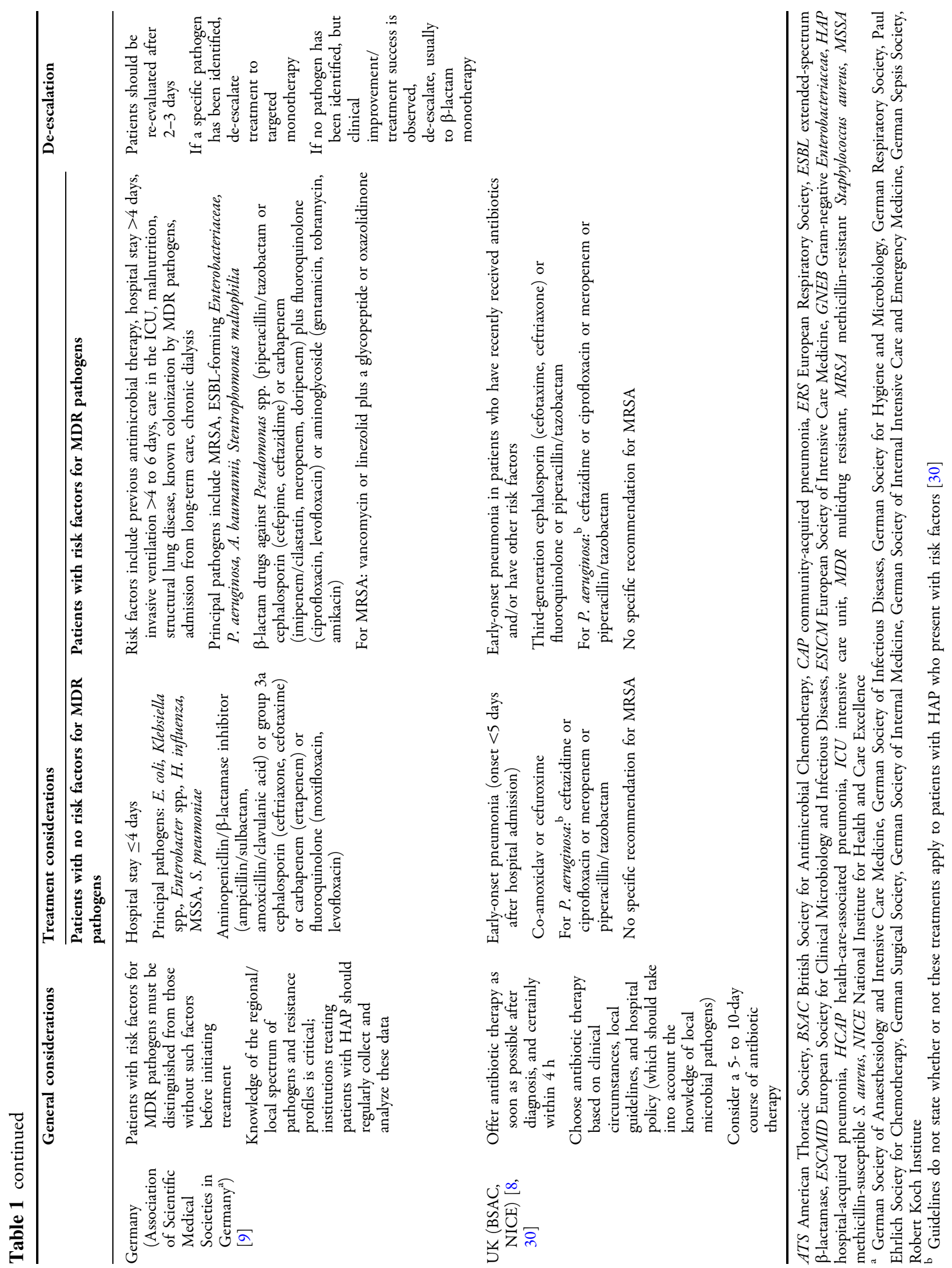


HAP) state that "the evidence base does not support the use of this term [HCAP] as being clinically relevant in Europe at the present time" [12]; similar doubts were also raised in the German guidelines published in 2013 [9].

Interestingly, the definition of HCAP used in clinical studies varies greatly, particularly regarding the length of time since previous hospital stay, the definition of and exposure to health-care facilities, and the inclusion/ exclusion of immunocompromised patients [7, $13,14]$. The heterogeneity of the studies of patients with HCAP may contribute to the uncertainty surrounding the acceptance of HCAP as a distinct subgroup of pneumonia.

\section{RISK FACTORS, ETIOLOGY, AND DIAGNOSIS OF HAP AND HCAP}

Individuals at the greatest risk of HAP include elderly people, patients who are immunocompromised or malnourished, patients with depression of consciousness, chronic renal failure, or multiple comorbidities (e.g., Charlson comorbidity index $\geq 3$ ), or those who have undergone surgery [2, 10]; increased length of hospital stay is also associated with a higher risk of developing HAP. Similar risk factors are documented for $\operatorname{HCAP}[7,15]$.

Etiologies of HAP and HCAP vary according to geographic area and risk factors. HAP typically involves infection with a single pathogen, such as a member of the Enterobacteriaceae (e.g., Klebsiella spp., Enterobacter spp., and Serratia spp.), Staphylococcus aureus (including methicillin-resistant $S$. aureus [MRSA]), Pseudomonas aeruginosa, or Acinetobacter baumannii [11, 16, 17]. HCAP is typically associated with pathogens such as Streptococcus pneumoniae, Klebsiella pneumoniae, and S. aureus $[14,18,19]$.

Questions remain regarding some of the characteristics of HCAP [20], such as its association with a high frequency of multidrug-resistant (MDR) pathogens [21-24] and the assumption that antibiotic treatment for CAP would be insufficient for patients with HCAP [21]. Indeed, several studies have found a greater prevalence of hard-to-treat or MDR pathogens in patients with HCAP than in those with CAP $[7,25,26]$. A study of 4543 patients in the USA found that $18.3 \%$ and $16.8 \%$ of patients with HCAP and HAP, respectively, were infected with MRSA, compared with $6.2 \%$ of those with CAP [7]. Furthermore, a study in 727 patients in Spain reported that penicillin-resistant pneumococcal strains were more commonly isolated from patients with HCAP than from those with CAP (33.3\% vs. $14.9 \%)$. In contrast, a low incidence of MDR pathogens was observed in both patients with HCAP and those with CAP in a separate study in Spain [22] and in a small UK study ( $n=138)$ [23].

Rapid identification of the specific causative pathogen(s) in patients with HAP and HCAP is essential to ensure that patients receive the most appropriate treatment as soon as possible. The identification of specific pathogens is challenging. The results of bacteriological assays are not always consistent and reproducible, and inaccurate cultures can result in failure to treat the relevant pathogens [2]. Moreover, invasive techniques for microbiological analysis are often difficult to perform on nonventilated individuals, and patients may be considered too ill for the procedure. In addition, specialized laboratories and training are required for many procedures and bronchoscopy may not be immediately available $[2,27]$. 
TREATMENT OF HAP AND HCAP: CURRENT GUIDELINES AND CONSIDERATIONS

The initial approach to the treatment of patients with HAP and HCAP is often necessarily empirical because complete results of antimicrobial susceptibility testing of the causative pathogens are typically available only after $48-72$ h. Appropriate empirical therapy should not be delayed, because inadequate and delayed treatment is associated with increased morbidity and mortality [28, 29]. Successful antimicrobial treatment depends on the use of an agent or regimen that is effective against the causative pathogen(s), and broad-spectrum therapy is often recommended [2]. Antimicrobial therapies that act rapidly reduce the length of hospital stays that in turn reduce resource utilization and health-care system costs [28].

\section{US and European Guidelines}

A summary of key recommendations from the major US and European HAP and HCAP guidelines is provided in Table $1[2,8,9,11$, $30]$. In the most recent ATS guidelines (2005) [2], treatment recommendations for HAP and HCAP are based on whether or not patients have risk factors for MDR pathogens, with more aggressive therapeutic strategies recommended for those who do; notably, all patients with HCAP are classified as being at risk of having MDR pathogens. Approaches to initial therapy provided in individual guidelines from Europe range from disease stage-specific antibiotic interventions to decision-making based on local conditions; however, as with the ATS recommendations, most guidelines categorize treatment strategies according to the risk of the presence of MDR bacteria [2, 30, 31]. Where this approach is employed, aminopenicillins with $\beta$-lactamase inhibitors, second- or third-generation cephalosporins, quinolones, or ertapenem are advocated for patients considered to be at low risk (e.g., early-onset HAP and/or no MDR pathogen risk). For patients thought to be at higher risk (e.g., late-onset HAP, MDR pathogen risk, in the ICU, and/or with organ failure), imipenem, meropenem, fourth-generation cephalosporins, ceftazidime, or piperacillin/tazobactam plus quinolones or aminoglycosides are recommended, depending on the degree of risk [32]. When MRSA is implicated in HAP, all guidelines recommend either linezolid or vancomycin (Table 1), but do not state a preference because direct comparisons have shown no difference in overall survival rate (although cure rates are higher with linezolid) [33]. It should also be emphasized that the therapeutic response is often poor if $P$. aeruginosa and MRSA are present, and infections with either of these pathogens are difficult to treat even when appropriate initial antibiotic therapy is used [34-36].

Joint European guidelines for the management of HAP recommend that treatment is selected according to the timing of disease onset (early onset $[\leq 4$ days of hospital admission] or late onset [ $>4$ days of hospital admission]) and whether risk factors are present (any onset) (Table 1) [11]. Where risk factors are present, the choice of therapy should reflect the suspected pathogen and take local bacterial spectrums into consideration. Once the pathogen responsible has been identified, therapy should focus on the relevant pathogen(s), and most patients should be switched to monotherapy after 3-5 days [11]. 


\section{National and Local Guidelines}

Treatment guidelines at the national level vary from general considerations and broad therapy suggestions to specific recommendations for different patient types and pathogens. Recent national guidelines highlight the importance of considering the spectrum of pathogens in the local area and their resistance profiles. The 2013 German guidelines categorize treatments for HAP based on whether or not patients have risk factors for the presence of MDR pathogens (Table 1) [9]. The guidelines advocate continuing treatment for 8 days, with de-escalation $48-72 \mathrm{~h}$ after the start of treatment when appropriate [9]. In contrast to these specific recommendations, UK guidelines for the management of HAP published in 2008 contain few definitive treatment recommendations (Table 1), citing lack of conclusive clinical evidence [30]. In December 2014, the UK National Institute for Health and Care Excellence (NICE) issued updated guidance beyond areas of care for which best practice was already established, recommending (i) the prompt initiation of therapy (certainly within $4 \mathrm{~h}$ ), (ii) that the choice of therapy should reflect local hospital policy, and (iii) that a 5- to 10-day course of therapy be considered (Table 1) [8].

Further and more detailed guidelines for the treatment of HAP are often issued at regional or local levels and even by individual hospitals [37, 38], although few of these are readily available. Recommendations at the local level tend to reflect differences in the spectrum of pathogens associated with HAP in the particular area. An example of this is the guidance related to MRSA, which has a higher prevalence in southern Europe than in northern Europe [39], potentially linked to increased antibiotic use in southern Europe [40]. Treatment strategies may also be tailored to local resistance patterns. For example, policies issued by the Royal Devon and Exeter NHS Foundation Trust (south-west UK) recommend vancomycin for all patients with moderate-to-severe HAP who are at high risk of MRSA infection [37], while those from Nottingham University Hospitals NHS Trust (central UK) note that nearly all $S$. aureus strains are sensitive to gentamicin and doxycycline, so linezolid and vancomycin are recommended only for patients in the ICU where there is known MRSA colonization [38].

\section{Further Considerations for Treatment Selection}

Guidelines are important for providing recommendations on which treatments (or classes of treatments) are appropriate for $\mathrm{HAP} /$ HCAP, but patient-specific considerations also influence treatment choice. These include duration of hospitalization before development of pneumonia, HAP staging, antimicrobial resistance patterns within the hospital, and previous antibiotic use [29]. Patient risk factors such as older age, presence of congestive heart failure, corticosteroid treatment, endotracheal intubation, neutropenia, and septic shock also affect the choice of therapy [28]. In addition, many patients with HAP or HCAP have comorbidities such as chronic renal failure that exclude them from receiving certain treatments $[7,41]$.

\section{Therapeutic Failure and De-escalation of Therapy}

Failure to respond to initial empirical antibiotic therapy can be expected in approximately $20-40 \%$ of patients with HAP, depending on the causative pathogen, the nature of the 
infection, and the presence and severity of comorbidities [11]. Therapeutic failure requires immediate and extensive diagnostic re-evaluation of the patient, including bronchoscopic respiratory secretion sampling and blood cultures [11, 42]. This should be accompanied by assessment of other complications, alternative diagnoses (e.g., atelectasis, congestive heart failure), and other sites of infection in the patient [2].

Effective de-escalation of broad-spectrum empirical therapy is important if patients' exposure to antibiotics and treatment resistance are to be minimized. The current European guidelines recommend that de-escalation begins once the pathogen has been identified (usually after 3-5 days; after 2-3 days in Germany) (Table 1) $[9,11]$. De-escalation can be conducted in several ways. First, therapy can focus on an antibiotic with a narrower spectrum of activity. In particular, vancomycin and linezolid treatment should be stopped if MRSA is not identified, and very broad-spectrum agents, such as carbapenems, piperacillin-tazobactam, and/or cefepime, should be restricted to patients infected with pathogens susceptible only to these agents [11]. Secondly, if the dosage of the initial antibiotic was high (e.g., based on pharmacodynamic optimization), it can be reduced to a standard dosage for a susceptible organism. Thirdly, if no pathogen is identified but clinical improvement has been observed, antibiotic therapy can be reduced (usually, to $\beta$-lactam monotherapy) or discontinued altogether [9, 11].

\section{NEW TREATMENT OPTIONS FOR HAP AND HCAP}

The persisting high mortality associated with HAP and HCAP $[5,7]$ indicates that current treatments are far from optimal. In addition to suboptimal efficacy, factors such as high nephrotoxicity rates (in $15.4 \%$ of patients with HAP treated with vancomycin) [43] and myelosuppression (associated with linezolid) [44] limit the use of certain antimicrobials. In addition, infections involving MRSA, mixed Gram-positive and Gram-negative bacteria, or MDR pathogens cannot currently be treated adequately by any single agent [5]. Hence, there remains a need for additional antibiotics that provide well-tolerated, broad-spectrum activity against MDR pathogens. Furthermore, alternative broad-spectrum antibiotics for HAP may offer carbapenem-sparing treatment options and thus help reduce the resistance selection pressure on carbapenems and limit the emergence of carbapenemases. The availability of such treatments would simplify the initial empirical therapy for HAP and HCAP.

In the 'golden age' of antibiotic development between 1935 and 1968, 14 classes of drug were introduced for human use. Since then, only five new classes have been added. Few new therapeutic agents for HAP have been approved recently. The most promising recent approvals include telavancin and ceftobiprole medocaril (Table 2) [45, 46]; tedizolid phosphate is currently being investigated for the treatment of CAP, HAP, VAP, and bacteremia.

\section{Telavancin}

Telavancin is a semisynthetic lipopolypeptide with approximately tenfold greater potency than vancomycin [47] and exclusively displays in vitro activity against clinically important Gram-positive bacteria including MRSA, vancomycin-intermediate S.aureus, and penicillin-resistant S.pneumoniae [47-50]. Bactericidal activity is concentration dependent and, with a half-life of 7-9 h, 


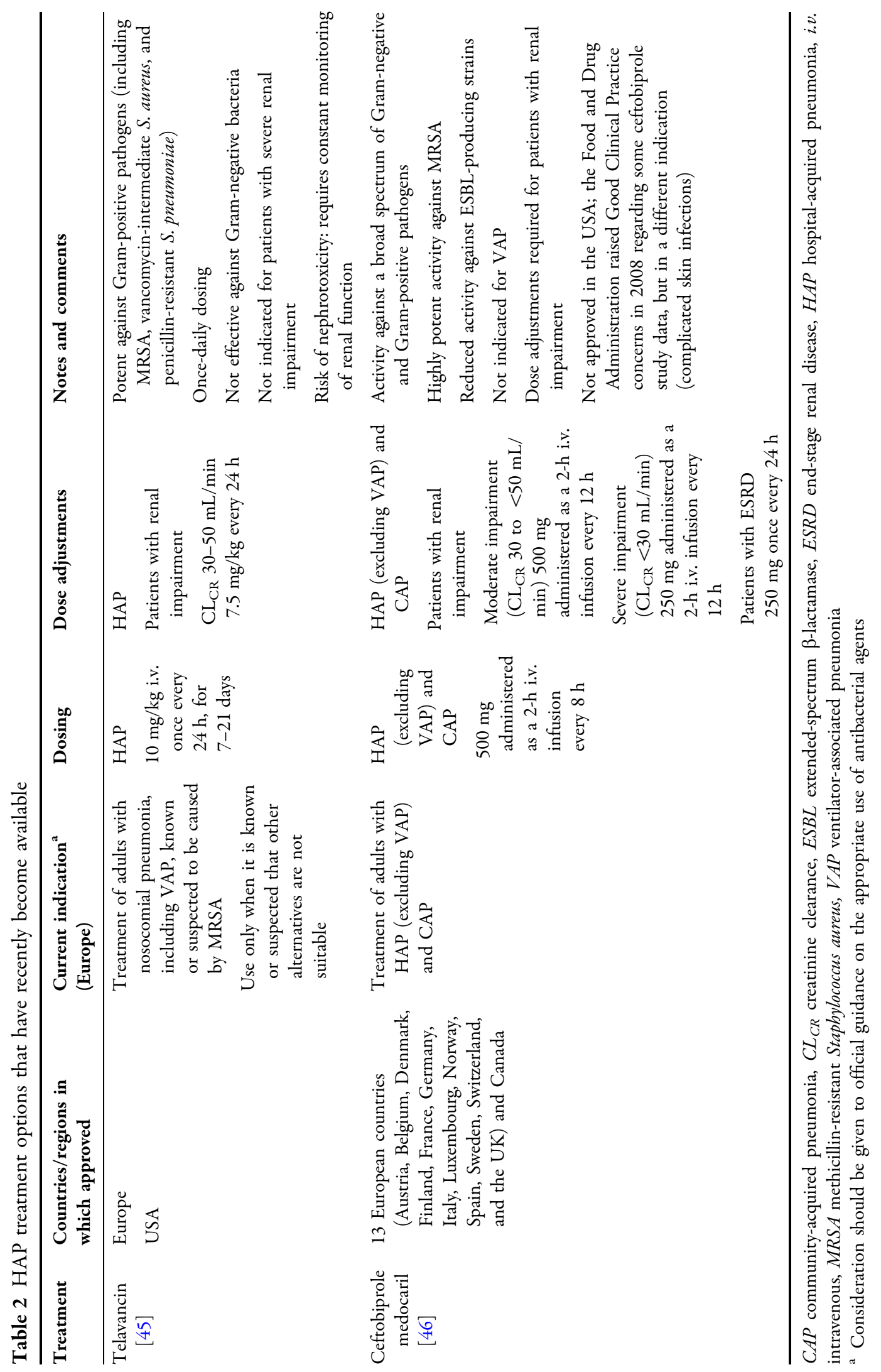


telavancin is suitable for once-daily dosing [51, 52]. On the basis of the results of the Assessment of Telavancin for Treatment of Hospital-Acquired Pneumonia (ATTAIN) studies, telavancin was approved in Europe (2011) and the USA (2013) for the treatment of HAP caused by Gram-positive pathogens including MRSA when alternative therapies are not suitable (Table 2) [45]. The two ATTAIN studies (ClinicalTrials.gov identifiers, NCT00107952 and NCT00124020) were double-blind, phase 3 clinical trials that assessed the efficacy and safety of telavancin in a total of 1503 patients with HAP caused by Gram-positive pathogens [53]. In the pooled all-treated population, intravenous (i.v.) telavancin $10 \mathrm{mg} / \mathrm{kg}$ every $24 \mathrm{~h}(n=749)$ was non-inferior to vancomycin $1 \mathrm{~g}$ i.v. every $12 \mathrm{~h}$ $(n=754)$ in terms of clinical cure rate at the follow-up/test-of-cure (TOC) visit [58.9\% vs. $59.5 \%$, respectively; $95 \%$ confidence interval (CI) for the difference $-5.6 \%, 4.3 \%]$. Moreover, in the subgroup of patients with $S$.aureus infection $(n=433)$, telavancin provided a higher clinical cure rate at the TOC visit than vancomycin $(78.1 \%$ vs. $75.2 \%$; $95 \%$ CI for the difference $-5.0 \%, 11.0 \%)$. Cure rates were similar in the subgroup of patients with MRSA infection $(n=293 ; 74.8 \%$ and $74.7 \%$ for telavancin and vancomycin, respectively; $95 \%$ CI for the difference $-9.5 \%, 10.4 \%$ ).

A post hoc analysis reported similar 28-day survival rates with telavancin and vancomycin (76\% and 77\%, respectively) [55], consistent with the mortality data from the original analyses [53]. However, lower survival rates were observed with telavancin compared with vancomycin (59\% vs. $70 \%)$ in patients with moderate-to-severe renal insufficiency (creatinine clearance $<50 \mathrm{~mL} / \mathrm{min}$ ) $[54,55]$; consequently, a black box warning for patients with inadequate renal function was added to the label in the USA. A subsequent post hoc analysis of the ATTAIN studies has suggested that the higher mortality rate for telavancin compared with vancomycin (telavancin, 179/751 patients; vancomycin, 163/752 patients) may have been confounded by inadequate coverage of Gram-negative infections, especially in the telavancin group [54]. Indeed, there were more patients with Gram-negative only infections and with inadequate treatment of those infections in the telavancin group than the vancomycin group. However, consistent with the black box warning, the post hoc analysis also found increased mortality with telavancin compared with vancomycin in patients with poor renal function (telavancin, 20/32 patients; vancomycin, $7 / 27$ patients [creatinine clearance $<30 \mathrm{~mL} / \mathrm{min}$ ]) [54].

Rates of treatment-emergent adverse events (AEs) were the same for telavancin and vancomycin (both 82\%); the most common AEs were diarrhea, anemia, hypokalemia, constipation, and renal impairment. The proportion of patients experiencing serious AEs was higher in the telavancin group than in the vancomycin group (31\% vs. $26 \%$ ). AEs leading to study discontinuation were also higher with telavancin than with vancomycin ( $8 \%$ vs. $5 \%)$; acute renal failure was the most common $\mathrm{AE}$ associated with discontinuation in the telavancin group (1.2\%) [53]. AEs associated with telavancin included transient elevations in serum creatinine levels, thrombocytopenia and QT prolongation, although to date no cardiovascular events attributed to QT prolongation have been reported [53, 56-58]. It is recommended that patients receiving telavancin are monitored closely (particularly, their renal function), owing to the associated AEs [45].

The potent activity of telavancin against Gram-positive HAP pathogens (including 
MRSA, vancomycin-intermediate $S$. aureus, and penicillin-resistant S.pneumoniae), and the convenient, once-daily dosing regimen may offer advantages over conventional therapies (Table 2). However, telavancin is not effective against Gram-negative bacteria [59]. In addition, it is not indicated for patients with severe renal impairment and the risk of nephrotoxicity necessitates constant monitoring of renal function [45].

\section{Ceftobiprole Medocaril}

Ceftobiprole medocaril, the prodrug of the active moiety ceftobiprole, is a new-generation, broad-spectrum i.v. cephalosporin. Ceftobiprole has unique activity against MRSA and a broad range of Gram-positive and Gram-negative pathogens [60], with high activity against methicillin-susceptible $S$. aureus, MRSA and S. pneumoniae (100\%, 98.3\%, and 99.3\% susceptibility, respectively) [61]. Interestingly, ceftobiprole appears to have high affinity for most penicillin-binding proteins (PBPs); in Escherichia coli, the major targets seem to be PBP1b and PBP2 rather than PBP3 (the target for third-generation cephalosporins) [62]. This PBP binding contributes to the potent activity of ceftobiprole against most Enterobacteriaceae spp. (87.3\% susceptibility); the activity of ceftobiprole against $P$. aeruginosa is similar to that of ceftazidime (64.6\% susceptibility) [61].

The efficacy of ceftobiprole in patients with HAP was assessed in a double-blind, randomized, controlled phase 3 study ( $n=781$, intent-to-treat [ITT] population, including 210 patients with VAP) with a dosing regimen of $500 \mathrm{mg}$ i.v. every $8 \mathrm{~h}$ (ClinicalTrials.gov identifiers: NCT00210964 and NCT00229008) [63]. Ceftobiprole was non-inferior to ceftazidime for clinical cure ( $2 \mathrm{~g}$ i.v. every $8 \mathrm{~h}$, plus linezolid $600 \mathrm{mg}$ i.v. twice daily) for both the whole population (ITT; $49.9 \%$ vs. $52.8 \%$, respectively) and the HAP-specific patient subgroup $(n=571 ; 59.6 \%$ vs. $58.8 \%$, respectively), although not in patients with VAP $(23.1 \%$ vs. $36.8 \%$, respectively) [63]. This difference in clinical cure rates was also evident when microbiologically evaluable $(n=332)$ patients were stratified according to baseline pathogen profile [63]. The microbiological eradication rates in patients with HAP (excluding VAP) were $62.9 \%$ vs. $67.5 \%$ in the ceftobiprole and ceftazidime plus linezolid groups, respectively; in patients with VAP, the microbiological eradication rates were $30.4 \%$ vs. $50.0 \%$, respectively. Overall, 30-day all-cause mortality and 30-day pneumonia-specific mortality were similar in the ceftobiprole and ceftazidime/linezolid treatment groups. Similar rates of treatment-related AEs were reported for ceftobiprole (24.9\%) and ceftazidime/linezolid $(25.4 \%)$ [63, 64]. Furthermore, in a randomized controlled trial in 638 patients requiring hospitalization for CAP (ClinicalTrials.gov identifier: NCT00326287), ceftobiprole $500 \mathrm{mg}$ i.v. twice daily was shown to be non-inferior to ceftriaxone $2 \mathrm{~g}$ i.v. once daily (with or without linezolid $600 \mathrm{mg}$ i.v. twice daily) for clinical cure [65].

Regulatory approval for ceftobiprole was obtained in October 2013 for the treatment of CAP and HAP, but not VAP, in 13 European countries (Table 2) [46, 66]. Ceftobiprole is also approved in Canada. At present, it is not approved in the USA (Table 2). The Food and Drugs Administration (FDA) raised some Good Clinical Practice concerns in 2008 over a number of ceftobiprole studies, but this was with regard to studies in complicated skin infections and did not affect those for pneumonia (HAP or CAP). Importantly, following inspections of the pneumonia 
clinical trial sites conducted by regulatory agencies during the European regulatory review, it was concluded that the data from these studies were reliable and represent a fair assessment of the efficacy and safety of ceftobiprole in these indications. Moreover, in August 2015, the FDA designated ceftobiprole as a Qualified Infectious Disease Product with potential use in the treatment of CAP and acute bacterial skin and skin structure infections.

The standard dose of ceftobiprole is $500 \mathrm{mg}$ every $8 \mathrm{~h}$; dose adjustment of ceftobiprole is recommended in patients with moderate or severe renal impairment [46]. In individuals with moderate impairment $\left(\mathrm{CL}_{\mathrm{CR}} 30\right.$ to $<50 \mathrm{~mL}$ / $\mathrm{min})$, the recommended dose is $500 \mathrm{mg}$ administered as a 2-h i.v. infusion every $12 \mathrm{~h}$; for those with severe impairment $\left(\mathrm{CL}_{\mathrm{CR}}<30 \mathrm{~mL} / \mathrm{min}\right)$ the recommended dose is $250 \mathrm{mg}$ administered as a 2-h i.v. infusion every $12 \mathrm{~h}$, and for patients with end-stage renal disease the recommended dose is $250 \mathrm{mg}$ once every $24 \mathrm{~h} \mathrm{[46].} \mathrm{There} \mathrm{is} \mathrm{no} \mathrm{need} \mathrm{for} \mathrm{dose}$ adjustment based on sex, ethnicity, age, or hepatic impairment [46].

\section{Tedizolid}

Tedizolid is a novel oxazolidinone antibiotic with potent activity against a wide range of Gram-positive pathogens, including MRSA and vancomycin-resistant enterococci $\left(\mathrm{MIC}_{90}\right.$ $0.25-0.5 \mu \mathrm{g} / \mathrm{mL}$ ) [67]. A phase 3, randomized, double-blind study comparing the efficacy and safety of tedizolid and linezolid is ongoing in patients with HAP and VAP, and is expected to be complete in February 2018 (ClinicalTrials.gov identifier: NCT02019420).

\section{CONCLUSION}

Both HAP and HCAP are common infections associated with high mortality and resource utilization. HCAP is not consistently recognized, but may be associated with an etiology more closely related to HAP than CAP. It is important to treat HAP and HCAP early with broad-spectrum antibiotics, because inadequate empirical therapy is associated with increased mortality and health-care costs. The current treatments for HAP and HCAP include all broad-spectrum antibiotics, but the burden of HAP and HCAP remains high. Additional treatment options have recently become available. Telavancin exhibits potent antibacterial activity against a range of Gram-positive bacteria, including MRSA, and has demonstrated non-inferiority to vancomycin in patients with HAP. The new-generation cephalosporin ceftobiprole shows rapid and potent antibacterial activity against a broad range of both Gram-positive (including MRSA) and Gram-negative pathogens important in HAP. It has demonstrated non-inferiority to combination therapy with ceftazidime and linezolid in patients with HAP (excluding VAP). These new agents may offer effective alternative options for the management of HAP.

\section{ACKNOWLEDGMENTS}

Medical writing assistance for this manuscript was provided by Dr Mark Rolfe and Dr Lizzy McAdam-Gray of Oxford PharmaGenesis, Oxford, UK; support for this assistance was funded by Basilea Pharmaceutica Ltd., Basel, 
Switzerland. All named authors meet the International Committee of Medical Journal Editors criteria for authorship for this manuscript, take responsibility for the integrity of the work as a whole, and have given final approval to the version to be published.

Disclosures. Philippe Montravers has been a speaker for Astellas, AstraZeneca, Basilea, Cubist, Gilead, MSD, Pfizer, and The Medicines Company, and an advisory board member for Astellas, AstraZeneca, Cubist, MSD, Pfizer, and The Medicines Company. Adela Harpan and Elise Guivarch have nothing to disclose.

Compliance with Ethics Guidelines. This article is based on previously conducted studies and does not involve any new studies of human or animal subjects performed by any of the authors.

Open Access. This article is distributed under the terms of the Creative Commons Attribution-NonCommercial 4.0 International License (http://creativecommons.org/licenses/ by-nc/4.0/), which permits any noncommercial use, distribution, and reproduction in any medium, provided you give appropriate credit to the original author(s) and the source, provide a link to the Creative Commons license, and indicate if changes were made.

\section{REFERENCES}

1. Chawla R. Epidemiology, etiology, and diagnosis of hospital-acquired pneumonia and ventilator-associated pneumonia in Asian countries. Am J Infect Control. 2008;36(Suppl 4):S93-100.

2. American Thoracic Society, Infectious Diseases Society of America. Guidelines for the management of adults with hospital-acquired, ventilator-associated, and healthcare-associated pneumonia. Am J Respir Crit Care Med. 2005;171(4):388-416.

3. Montravers P, Veber B, Auboyer C, et al. Diagnostic and therapeutic management of nosocomial pneumonia in surgical patients: results of the Eole study. Crit Care Med. 2002;30(2):368-75.

4. Kollef $\mathrm{MH}$, Hamilton $\mathrm{CW}$, Ernst FR. Economic impact of ventilator-associated pneumonia in a large matched cohort. Infect Control Hosp Epidemiol. 2012;33(3):250-6.

5. Barbier F, Andremont A, Wolff M, Bouadma L. Hospital-acquired pneumonia and ventilator-associated pneumonia: recent advances in epidemiology and management. Curr Opin Pulm Med. 2013;19(3):216-28.

6. Chastre J, Blasi F, Masterton RG, Rello J, Torres A, Welte T. European perspective and update on the management of nosocomial pneumonia due to methicillin-resistant Staphylococcus aureus after more than 10 years of experience with linezolid. Clin Microbiol Infect. 2014;20(Suppl 4):19-36.

7. Kollef MH, Shorr A, Tabak YP, Gupta V, Liu LZ, Johannes RS. Epidemiology and outcomes of health-care-associated pneumonia: results from a large US database of culture-positive pneumonia. Chest. 2005;128(6):3854-62.

8. National Institute for Health and Care Excellence (NICE). Pneumonia: Diagnosis and management of community- and hospital-acquired pneumonia in adults 2014 [updated December 2014; cited CG191]. http://publications.nice.org.uk/ pneumonia-cg191/recommendations\#terms-usedin-this-guideline. Accessed Nov 2015.

9. Dalhoff K, Ewig S. Adult patients with nosocomial pneumonia: epidemiology, diagnosis, and treatment. Dtsch Arztebl Int. 2013;110(38):634-40.

10. Sopena N, Heras E, Casas I, et al. Risk factors for hospital-acquired pneumonia outside the intensive care unit: a case-control study. Am J Infect Control. 2014;42(1):38-42.

11. Torres A, Ewig S, Lode H, Carlet J. Defining, treating and preventing hospital acquired pneumonia: European perspective. Intensive Care Med. 2009;35(1):9-29.

12. Woodhead M, Blasi F, Ewig S, et al. Guidelines for the management of adult lower respiratory tract infections-full version. Clin Microbiol Infect. 2011;17(Suppl 6):E1-59.

13. Ewig S, Welte T, Chastre J, Torres A. Rethinking the concepts of community-acquired and 
health-care-associated pneumonia. Lancet Infect Dis. 2010;10(4):279-87.

14. Micek ST, Kollef KE, Reichley RM, Roubinian N, Kollef MH. Health care-associated pneumonia and community-acquired pneumonia: a single-center experience. Antimicrob Agents Chemother. 2007;51(10):3568-73.

15. Friedman ND, Kaye KS, Stout JE, et al. Health care-associated bloodstream infections in adults: a reason to change the accepted definition of community-acquired infections. Ann Intern Med. 2002;137(10):791-7.

16. Allou N, Kermarrec N, Muller C, et al. Risk factors and prognosis of post-operative pneumonia due to Pseudomonas aeruginosa following cardiac surgery. J Antimicrob Chemother. 2010;65(4):806-7.

17. Koulenti D, Lisboa T, Brun-Buisson C, et al. Spectrum of practice in the diagnosis of nosocomial pneumonia in patients requiring mechanical ventilation in European intensive care units. Crit Care Med. 2009;37(8):2360-8.

18. Jung JY, Park MS, Kim YS, et al. Healthcare-associated pneumonia among hospitalized patients in a Korean tertiary hospital. BMC Infect Dis. 2011;11:61.

19. Shindo Y, Sato S, Maruyama E, et al. Health-care-associated pneumonia among hospitalized patients in a Japanese community hospital. Chest. 2009;135(3):633-40.

20. Ewig S, Welte T, Torres A. Is healthcare-associated pneumonia a distinct entity needing specific therapy? Curr Opin Infect Dis. 2012;25(2):166-75.

21. Dobler CC, Waterer G. Healthcare-associated pneumonia: a US disease or relevant to the Asia Pacific, too? Respirology. 2013;18(6):923-32.

22. Garcia-Vidal C, Viasus D, Roset A, et al. Low incidence of multidrug-resistant organisms in patients with healthcare-associated pneumonia requiring hospitalization. Clin Microbiol Infect. 2011;17(11):1659-65.

23. Chalmers JD, Taylor JK, Singanayagam A, et al. Epidemiology, antibiotic therapy, and clinical outcomes in health care-associated pneumonia: a UK cohort study. Clin Infect Dis. 2011;53(2):107-13.

24. Chalmers JD, Rother C, Salih W, Ewig S. Healthcare-associated pneumonia does not accurately identify potentially resistant pathogens: a systematic review and meta-analysis. Clin Infect Dis. 2014;58(3):330-9.
25. Shindo $\mathrm{Y}$, Ito R, Kobayashi D, et al. Risk factors for drug-resistant pathogens in community-acquired and healthcare-associated pneumonia. Am J Respir Crit Care Med. 2013;188(8):985-95.

26. Carratala J, Mykietiuk A, Fernandez-Sabe N, et al. Health care-associated pneumonia requiring hospital admission: epidemiology, antibiotic therapy, and clinical outcomes. Arch Intern Med. 2007;167(13):1393-9.

27. Rotstein C, Evans G, Born A, et al. Clinical practice guidelines for hospital-acquired pneumonia and ventilator-associated pneumonia in adults. Can J Infect Dis Med Microbiol. 2008;19(1):19-53.

28. Davey PG, Marwick C. Appropriate vs. inappropriate antimicrobial therapy. Clin Microbiol Infect. 2008;14(Suppl 3):15-21.

29. Lynch JP 3rd. Hospital-acquired pneumonia: risk factors, microbiology, and treatment. Chest. 2001;119(Suppl 2):373S-84S.

30. Masterton RG, Galloway A, French G, et al. Guidelines for the management of hospital-acquired pneumonia in the UK: report of the working party on hospital-acquired pneumonia of the British Society for Antimicrobial Chemotherapy. J Antimicrob Chemother. 2008;62(1):5-34.

31. Dalhoff K, Abele-Horn $\mathrm{M}$, Andreas $\mathrm{S}$, et al. Epidemiology, diagnosis and treatment of adult patients with nosocomial pneumonia. S-3 Guideline of the German Society for Anaesthesiology and Intensive Care Medicine, the German Society for Infectious Diseases, the German Society for Hygiene and Microbiology, the German Respiratory Society and the Paul-Ehrlich-Society for Chemotherapy. Pneumologie. 2012;66(12):707-65.

32. Wilke M, Grube R. Update on management options in the treatment of nosocomial and ventilator assisted pneumonia: review of actual guidelines and economic aspects of therapy. Infect Drug Resist. 2013;7:1-7.

33. Wunderink RG, Niederman MS, Kollef MH, et al. Linezolid in methicillin-resistant Staphylococcus aureus nosocomial pneumonia: a randomized, controlled study. Clin Infect Dis. 2012;54(5):621-9.

34. Lode $H$. Combination versus monotherapy for nosocomial pneumonia. Eur Respir Rev. 2007;16(103):50-5.

35. Kollef MH, Sherman G, Ward S, Fraser VJ. Inadequate antimicrobial treatment of infections: a risk factor for hospital mortality among critically ill patients. Chest. 1999;115(2):462-74. 
36. Vidaur L, Planas $\mathrm{K}$, Sierra $\mathrm{R}$, et al. Ventilator-associated pneumonia: impact of organisms on clinical resolution and medical resources utilization. Chest. 2008;133(3):625-32.

37. Royal Devon and Exeter hospital guidelines for the management of HAP. http://www.rdehospital.nhs. uk/docs/prof/antimicrobial/hap_sept2012_final_v_ fromv8_abxtables_approved.pdf. Accessed Nov 11 2015.

38. Nottingham University Hospital HAP treatment guidelines. http://www.nuh.nhs.uk/handlers/ downloads.ashx?id=60995. Accessed 11 Nov 2015.

39. Summary of the latest data on antibiotic resistance in the European Union. http://ecdc.europa.eu/en/ publications/Documents/antibiotic-resistance-inEU-summary.pdf. Accessed 13 Nov 2015.

40. MacKenzie FM, Bruce J, Struelens MJ, et al. Antimicrobial drug use and infection control practices associated with the prevalence of methicillin-resistant Staphylococcus aureus in European hospitals. Clin Microbiol Infect. 2007; 13(3):269-76.

41. Quartin AA, Scerpella EG, Puttagunta S, Kett DH. A comparison of microbiology and demographics among patients with healthcare-associated, hospital-acquired, and ventilator-associated pneumonia: a retrospective analysis of 1184 patients from a large, international study. BMC Infect Dis. 2013;13:561.

42. Pereira Gomes JC, Pedreira WL Jr, Araujo EM, et al. Impact of BAL in the management of pneumonia with treatment failure: positivity of BAL culture under antibiotic therapy. Chest. 2000;118(6): 1739-46.

43. Cano EL, Haque NZ, Welch VL, et al. Incidence of nephrotoxicity and association with vancomycin use in intensive care unit patients with pneumonia: retrospective analysis of the IMPACT-HAP Database. Clin Ther. 2012;34(1):149-57.

44. Anger HA, Dworkin F, Sharma S, Munsiff SS, Nilsen DM, Ahuja SD. Linezolid use for treatment of multidrug-resistant and extensively drug-resistant tuberculosis, New York City, 2000-06. J Antimicrob Chemother. 2010;65(4):775-83.

45. Clinigen Healthcare Ltd. VIBATIV $250 \mathrm{mg}$ powder for concentrate for solution for infusion (SmPC) 2015. http://www.medicines.org.uk/emc/medicine/ 29035. Accessed 13 Nov 2015.

46. Basilea Pharmaceutica International Ltd. Zevtera $500 \mathrm{mg}$ powder for concentrate for solution for infusion (SmPC) 2015. https://www.medicines.org. uk/emc/medicine/29764. Accessed 13 Nov 2015.
47. Mendes RE, Sader HS, Farrell DJ, Jones RN. Worldwide appraisal and update (2010) of telavancin activity tested against a collection of Gram-positive clinical pathogens from five continents. Antimicrob Agents Chemother. 2012;56(7):3999-4004.

48. Leuthner KD, Cheung CM, Rybak MJ. Comparative activity of the new lipoglycopeptide telavancin in the presence and absence of serum against 50 glycopeptide non-susceptible staphylococci and three vancomycin-resistant Staphylococcus aureus. J Antimicrob Chemother. 2006;58(2):338-43.

49. Gotfried MH, Shaw JP, Benton BM, et al. Intrapulmonary distribution of intravenous telavancin in healthy subjects and effect of pulmonary surfactant on in vitro activities of telavancin and other antibiotics. Antimicrob Agents Chemother. 2008;52(1):92-7.

50. Pace JL, Krause K, Johnston D, et al. In vitro activity of TD-6424 against Staphylococcus aureus. Antimicrob Agents Chemother. 2003;47(11):3602-4.

51. Hegde SS, Reyes N, Wiens $\mathrm{T}$, et al. Pharmacodynamics of telavancin (TD-6424), a novel bactericidal agent, against gram-positive bacteria. Antimicrob Agents Chemother. 2004;48(8):3043-50.

52. Shaw JP, Seroogy J, Kaniga K, Higgins DL, Kitt M, Barriere S. Pharmacokinetics, serum inhibitory and bactericidal activity, and safety of telavancin in healthy subjects. Antimicrob Agents Chemother. 2005;49(1):195-201.

53. Rubinstein E, Lalani T, Corey GR, et al. Telavancin versus vancomycin for hospital-acquired pneumonia due to gram-positive pathogens. Clin Infect Dis. 2011;52(1):31-40.

54. Lacy MK, Stryjewski ME, Wang W, et al. Telavancin hospital-acquired pneumonia trials: impact of Gram-negative infections and inadequate Gram-negative coverage on clinical efficacy and all-cause mortality. Clin Infect Dis. 2015;61(Suppl 2):S87-93.

55. Corey GR, Kollef MH, Shorr AF, et al. Telavancin for hospital-acquired pneumonia: clinical response and 28-day survival. Antimicrob Agents Chemother. 2014;58(4):2030-7.

56. Stryjewski ME, Graham DR, Wilson SE, et al. Telavancin versus vancomycin for the treatment of complicated skin and skin-structure infections caused by gram-positive organisms. Clin Infect Dis. 2008;46(11):1683-93.

57. Stryjewski ME, Lentnek A, O'Riordan W, et al. A randomized Phase 2 trial of telavancin versus standard therapy in patients with uncomplicated 
Staphylococcus aureus bacteremia: the ASSURE study. BMC Infect Dis. 2014;14:289.

58. Stryjewski ME, O'Riordan WD, Lau WK, et al. Telavancin versus standard therapy for treatment of complicated skin and soft-tissue infections due to gram-positive bacteria. Clin Infect Dis. 2005;40(11):1601-7.

59. Jones RN. Microbial etiologies of hospital-acquired bacterial pneumonia and ventilator-associated bacterial pneumonia. Clin Infect Dis. 2010;51(Suppl 1):S81-7.

60. Syed YY. Ceftobiprole medocaril: a review of its use in patients with hospital- or community-acquired pneumonia. Drugs. 2014;74(13):1523-42.

61. Farrell DJ, Flamm RK, Sader HS, Jones RN. Ceftobiprole activity against over 60,000 clinical bacterial pathogens isolated in Europe, Turkey, and Israel from 2005 to 2010. Antimicrob Agents Chemother. 2014;58(7):3882-8.

62. Bustos C, Del Pozo JL. Emerging agents to combat complicated and resistant infections: focus on ceftobiprole. Infect Drug Resist. 2010;3:5-14.

63. Awad SS, Rodriguez AH, Chuang YC, et al. A Phase 3 randomized double-blind comparison of ceftobiprole medocaril versus ceftazidime plus linezolid for the treatment of hospital-acquired pneumonia. Clin Infect Dis. 2014;59:51-61.

64. Holmes NE, Tong SY, Davis JS, Hal SJ. Treatment of methicillin-resistant Staphylococcus aureus: vancomycin and beyond. Semin Respir Crit Care Med. 2015;36(1):17-30.

65. Nicholson SC, Welte T, File TM Jr, et al. A randomised, double-blind trial comparing ceftobiprole medocaril with ceftriaxone with or without linezolid for the treatment of patients with community-acquired pneumonia requiring hospitalisation. Int $\mathrm{J}$ Antimicrob Agents. 2012;39(3):240-6.

66. Basilea Pharmaceutica Ltd. Basilea's antibiotic ceftobiprole obtains regulatory approval in Europe for pneumonia 2013 [cited 2015 July]. http://www. basilea.com/chameleon/public/584f9d1e-4298-e47c0475-a5e5e5288ded/582542. Accessed 13 Nov 2015.

67. Locke JB, Zurenko GE, Shaw KJ, Bartizal K. Tedizolid for the management of human infections: in vitro characteristics. Clin Infect Dis. 2014;58(Suppl 1):S35-42. 